\title{
STRUCTURE OF REFINABLE SPLINES
}

\author{
XIN-RONG DAI, DE-JUN FENG, AND YANG WANG
}

\begin{abstract}
A refinable spline is a compactly supported refinable function that is piecewise polynomial. Refinable splines, such as the well known $B$-splines, play a key role in computer aided geometric designs. Refinable splines have been studied in several papers, most noticably in [7] for integer dilations and [3] for real dilations. There are general characterizations in these papers, but these characterizations are not explicit enough to tell us the structures of refinable splines. In this paper, we give complete characterization of the structure of refinable splines.
\end{abstract}

\section{INTRODUCTION}

A compactly supported real function $f(x)$ on $\mathbb{R}$ with $\operatorname{supp}(f)=[a, b]$ is called a spline if there exist $a=x_{0}<x_{1}<\cdots<x_{L}=b$ and polynomials $P_{j}(x)$ such that $f(x)=P_{j}(x)$ on $\left[x_{j-1}, x_{j}\right)$ for $1 \leq j \leq L$. In other words, a spline is a compactly supported piecewise polynomial function. Notice that we do not assume a spline is continuous. The points $\left\{x_{j}\right\}$ are called the knots of the spline, and $\max \left\{\operatorname{deg}\left(P_{j}\right)\right\}$ is called the degree of the spline.

Splines can have diverse properties. Among the most useful ones are those that are also refinable. A compactly supported function $f(x)$ is refinable if it satisfies a refinement equation

$$
f(x)=\sum_{k \in \mathbb{Z}} c_{k} f(\lambda x-k), \quad \sum_{k \in \mathbb{Z}} c_{k}=|\lambda|,
$$

where all $c_{k} \in \mathbb{R}$ with only finitely many $c_{k} \neq 0$, and $\lambda$ is real with $|\lambda|>1$ We call $\lambda$ the dilation factor or simply the dilation for the refinable function $f(x)$. It should be pointed out that $\lambda$ is not necessarily an integer, since there are refinable splines with non-integer dilations, see Dai, Feng and Wang [3]. It is also possible to allow non-integer translations, but we shall not discuss it here.

\footnotetext{
The second author is supported in part by the Special Funds for Major State Basic Research Projects in China.

The second author is supported in part by the National Science Foundation, grants DMS-0070586 and DMS-0139261.
} 
This paper studies the structure of refinable splines. Refinable functions and splines are among the most important functions, used extensively in applications such as numerical solutions to differential and integral equations, digital signal processing, image compression, and many others. Refinable functions form the foundation for the theory of compactly supported wavelets and the theory of subdivision schemes. There is a vast literature on both subjects. We refer the readers to Daubechies [5] and Cavaretta, Dahmen and Micchelli [1] as well as other sources for more details. Refinable splines such as the B-splines are the cornerstones in computer aided geometric designs. We aim to characterize the structure of compactly supported refinable splines in this paper.

Note that any translate of a refinable function is still refinable. Suppose that $f(x)$ satisfies (1.1) then $g(x)=f\left(x-\frac{b}{\lambda-1}\right)$ satisfies the refinement equation

$$
g(x)=\sum_{j=0}^{n} c_{j} g\left(\lambda x-d_{j}+b\right),
$$

which has the same dilation but a new translation set $\left\{d_{j}-b\right\}$.

Definition 1.1. A normalized refinement equation in $\mathbb{R}$ is a refinement equation having the form

$$
f(x)=\sum_{k=0}^{n} c_{k} f(\lambda x-k), \quad \sum_{k=0}^{n} c_{k}=|\lambda|,
$$

where $c_{0} \neq 0$. In this case we say $f(x)$ is normalized $\lambda$-refinable.

Thus every refinable function can be translated to satisfy a normalized refinement equation. We shall, without loss of generality, state our classification results for normalized refinable splines, since the statements are often more concise.

The simplest refinable spline is the Haar function $B_{0}(x)=\chi_{[0,1)}(x)$, which satisfies the refinement equation $f(x)=f(2 x)+f(2 x-1)$. In fact $B_{0}$ is $m$-refinable for any integer $m>1$, as

$$
B_{0}(x)=\sum_{j=0}^{m-1} B_{0}(m x-j) .
$$

It is easily checked that the convolution of $\lambda$-refinable functions (resp. spline) remains a $\lambda$-refinable function (resp. spline). Thus $B_{k}:=B_{0} * B_{0} * \cdots * B_{0}$ where $B_{0}$ convolves with itself $k$ times is also an $m$-refinable spline. The spline $B_{k}$ is known as the $B$-spline of degree 
$k$, which has knots at $0,1, \cdots, k+1$ and is $k-1$ times differentiable. In the case $m=2$, $B_{k}$ satisfies

$$
B_{k}(x)=\frac{1}{2^{k}} \sum_{j=0}^{k+1}\left(\begin{array}{c}
k+1 \\
j
\end{array}\right) B_{k}(2 x-j) .
$$

The following theorem lists several ways for characterizing (normalized) refinable splines.

Theorem 1.1. Let $f(x)$ be normalized $\lambda$-refinable with $\lambda \in \mathbb{Z}, \lambda>1$. Then the following are equivalent:

(A) $f(x)$ is a spline of degree $d \geq 0$.

(B) There exists a trigonometric polynomial $G(\xi)$ such that $\widehat{f}(\xi)=\xi^{-d-1} G(\xi)$.

(C) $f(x)=\sum_{k=0}^{N} p_{k} B_{d}(x-k)$ for some $N \geq 0$, and $\left\{p_{k}\right\} \subset \mathbb{R}$ such that the polynomial $q(z)=\left(\sum_{k=0}^{N} p_{k} z^{k}\right)(z-1)^{d+1}$ satisfies $q(z) \mid q\left(z^{\lambda}\right)$.

Theorem 1.1 is a combination of results in [7] and [3]. Although it is a nice general characterization, it falls short of giving a complete classification of the structure of refinable splines. The purpose of this paper is to complete the classification of refinable splines.

A refinable spline may have a non-integer dilation factor. However, the following theorem proved in Dai, Feng and Wang [3] states that a complete classfication of refinable splines with integer dilations are all we need to do.

Theorem $1.2([3])$. Suppose that $f(x)$ is a compactly supported spline satisfying the refinable equation (1.1), where $\lambda \in \mathbb{R}$. Then $f(x)$ is symmetric and $d-1$ times continuously differentiable. Furthermore,

(A) There exists an integer $k>0$ such that $\lambda^{k} \in \mathbb{Z}$.

(B) Let $K$ be the smallest positive integer such that $\lambda^{K} \in \mathbb{Z}$. Then the compactly supported distribution solution $\phi(x)$ to the refinement equation

$$
\phi(x)=\sum_{k \in \mathbb{Z}} c_{k} \phi(\lambda x-k)
$$

is a spline.

(C) There exists a constant $\alpha$ such that the spline $f(x)$ has

$$
f(x)=\alpha \phi(x) * \phi\left(\lambda^{-1} x\right) * \cdots * \phi\left(\lambda^{-(K-1)} x\right) .
$$

$\phi$ is the spline given in (1.4). 
Conversely, if the refinement equation (1.1) satisfies (A) and (B) then the compactly supported distribution solution is a spline given by (1.4).

With Theorem 1.2 we shall focus entirely on refinable splines with integer dilation factors in the rest of the paper.

So far all the examples of refinable splines we have seen have rational coefficients in the corresponding refinement equations. But in fact it does not have to be the case. In $\S 2$ we state our classfication results for both rational and general refinable splines. The results will then be proved in $\S 3$.

\section{Complete Classification of Refinable Splines in $\mathbb{R}$}

We first introduce some notations. Associated to the refinement equation (1.1) is a (Laurent) polynomial $h(z):=\frac{1}{|\lambda|} \sum_{k \in \mathbb{Z}} c_{k} z^{k}$ with $h(1)=1$. We shall call $h(z)$ the mask polynomial of the refinement equation (1.1). For normalized refinement equations $h(z)$ is a polynomial with nonzero constant term. In most studies on refinement equations the trigonometric polynomial $H(\xi):=h\left(e^{-2 \pi i \xi}\right)$ is used instead, and $H(\xi)$ is called the mask of the refinement equation. It is more convenient in this paper to work with the mask polynomial $h(z)$. A refinable spline is rational if it satisfies a rational refinement equation, i.e. all coefficients are rational.

There are two ways we can classify all normalized $\lambda$-refinable splines (with $\lambda \in \mathbb{Z}$ ): By classifying all mask polynomials $h(z)$ that lead to refinable splines, or by classifying all polynomials $q(z)=(z-1)^{d+1} \sum_{n} p_{n} z^{n}$ in part (C) of Theorem 1.1, which leads to the structure of the splines $f(x)=\sum_{n} p_{n} B_{d}(x-n)$. Depending on the situation, one might be more elegant than the other.

Also throughout this paper we let $\mathbb{Z}^{+}$denote the set of all positive integers. For any integer $m \in \mathbb{Z}^{+}$let $\Phi_{m}(z)$ denote the cyclotomic polynomial of order $m$, i.e. the monic polynomial whose roots comprise of primitive $m$-th roots of unity. We note that $\Phi_{1}(z)=$ $z-1$ and if $m$ is a prime then $\Phi_{m}(z)=z^{m-1}+z^{m-2}+\cdots+z+1$ with $\Phi_{m^{\alpha}}(z)=\Phi_{m}\left(z^{m^{\alpha-1}}\right)$. It is well known (see e.g. [2]) that $\Phi_{m}(0)=1$ for all $m>1, \Phi_{m}(1)=1$ if $m>1$ is not a prime power, and $\Phi_{m}(1)=p$ if $m=p^{\alpha}$ for some prime $p$ and $\alpha>0$.

Theorem 2.1. Let $\lambda$ be a prime. Then the following are equivalent: 
(a) $f(x)$ is a normalized rational $\lambda$-refinable spline of degree $d$.

(b) $f(x)$ satisfies a $\lambda$-refinement equation whose mask polynomial is

$$
h(z)=\lambda^{-d-1} \prod_{k=1}^{m} \Phi_{b_{k}}(z),
$$

where $\lambda \mid b_{k}$ for all $k$ and $d+1=\mid\left\{k: b_{k}=\lambda^{l}\right.$ for some positive interger $\left.l\right\} \mid$.

(c) $f(x)=\sum_{n=0}^{N} p_{n} B_{d}(x-n)$ with $p_{0} \neq 0$, for which

$$
q(z)=(z-1)^{d+1}\left(\sum_{n \geq 0} p_{n} z^{n}\right)=p_{0} \prod_{k=1}^{m} \prod_{j=0}^{\alpha_{k}-1} \Phi_{\lambda^{j} a_{k}}(z)
$$

for some $a_{k}, \alpha_{k} \in \mathbb{Z}^{+}$such that $\operatorname{gcd}\left(\lambda, a_{k}\right)=1$ for all $k$ and $d+1=\left|\left\{k: a_{k}=1\right\}\right|$.

When $\lambda>0$ is not a prime the characterization is more complex, especially for the mask polynomial $h(z)$. Let $m, n \in \mathbb{Z}^{+}$. Assume that $m, n$ have prime factorizations $m=\prod_{p} p^{\alpha_{p}}$ and $n=\prod_{p} p^{\beta_{p}}$, where $p$ runs through all primes and $\alpha_{p}, \beta_{p} \geq 0$. We define

$$
\langle m / n\rangle:=\prod_{p} p^{\gamma_{p}}, \quad \text { where } \gamma_{p}=\max \left\{\alpha_{p}-\beta_{p}, 0\right\} .
$$

Theorem 2.2. Let $\lambda>1$ be an integer. Then the following are equivalent:

(a) $f(x)$ is a normalized rational $\lambda$-refinable spline of degree $d$.

(b) $f(x)=\sum_{n=0}^{N} p_{n} B_{d}(x-n)$ with $p_{0} \neq 0$, for which

$$
q(z):=(z-1)^{d+1} \sum_{n=0}^{N} p_{n} z^{n}=p_{0} \prod_{k=1}^{m} \Phi_{b_{k}}^{\alpha_{k}}(z) .
$$

where $b_{k}, \alpha_{k} \in \mathbb{Z}^{+}, b_{1}=1, \alpha_{1}=d+1$, and $b_{k}$ 's are distinct. Furthermore, for any $k$ there exists some $j$ such that $b_{j}=\left\langle b_{k} / \lambda\right\rangle$ with the property that $\alpha_{j} \geq \alpha_{k}$.

Under the hypothesis of (b), $f(x)$ satisifies the $\lambda$-refinement equation with mask polynomial $h(z)=\lambda^{-d-1} q\left(z^{\lambda}\right) / q(z)$.

When the rationality assumption is dropped we can still characterize all refinable splines.

Theorem 2.3. Let $\lambda>1$ be an integer. Then the following are equivalent:

(a) $f(x)$ is a normalized $\lambda$-refinable spline of degree $d$.

(b) $f(x)=\sum_{n=0}^{N} p_{n} B_{d}(x-n)$ with $p_{0} \neq 0, \sum_{n=0}^{N} p_{n} \neq 0$, such that all roots of $q_{1}(z)=$ $\sum_{n=0}^{N} p_{n} z^{n}$ are roots of unity. Furthermore, if $\omega$ is a root of $q_{1}(z)$ with multiplicity 
$\alpha$ then either $\omega^{\lambda}=1$ with $\alpha \leq d+1$, or $\omega^{\lambda}$ is also a root of $q_{1}(z)$ with multiplicity at least $\alpha$.

Under the hypothesis of $(\mathrm{b}), f(x)$ satisifies the $\lambda$-refinement equation with mask polynomial $h(z)=\lambda^{-d-1} q\left(z^{\lambda}\right) / q(z)$, where $q(z)=(z-1)^{d+1} q_{1}(z)$.

Theorem 2.3 allows us to easily find $\lambda$-refinable splines $f(x)=\sum_{n=0}^{N} p_{n} B_{d}(x-n)$ by constructing the roots of $\sum_{n=0}^{N} p_{n} z^{n}$. In $\S 3$ we show an example of a refinable spline that is not rational. Another application of this theorem is to study functions functions that are $\lambda$-refinable for more than one dilations $\lambda$. As mentioned in the introduction, the box spline $B_{d}(x)$ is $\lambda$-refinable with respect to any integer dilation. Are there other such refianble functions? Similar questions have been asked in Sun and Zhang [8]. We answer this question here:

Theorem 2.4. A function $f(x)$ is normalized $\lambda$-refinable for all $\lambda \in \mathbb{Z}^{+}, \lambda>1$, if and only if there exist $N_{0}, N_{2}, \ldots, N_{d} \in \mathbb{Z}^{+}$such that $f(x)=c \chi_{\left[0, N_{0}\right)} * \chi_{\left[0, N_{1}\right)} * \cdots * \chi_{\left[0, N_{d}\right)}$ where $c \in \mathbb{R}, c \neq 0$.

Finally, one may have noticed that so far we have assumed that $\lambda>0$. What if $\lambda<0$ ? This is not an obstacle at all, due to the fact that a refinable spline is symmetric. If $f(x)$ is a $\lambda$-refinable spline then it is also a $(-\lambda)$-refinable spline. In fact, we have the following result:

Proposition 2.5. Assume that $f(x)$ is a refinable spline satisfying the refinement equation $f(x)=\sum_{k \in \mathbb{Z}} c_{k} f(\lambda x-k)$ with $\sum_{k \in \mathbb{Z}} c_{k}=|\lambda|$. Then for some $a \in \mathbb{R}, g(x)=f(x-a)$ satisfies the refinement equation $g(x)=\sum_{k \in \mathbb{Z}} c_{k} g(-\lambda x-k)$.

\section{Proof of Theorems}

Lemma 3.1. Let $f(x)=\sum_{n=0}^{N} p_{n} B_{d}(x-n), p_{0} \neq 0$ and $q(z)=(z-1)^{d+1} \sum_{n=0}^{N} p_{n} z^{n}$. Let $\lambda \in \mathbb{Z}, \lambda>1$. Assume that $f(x)$ is $\lambda$-refinable with mask polynomial $h(z)$. Then

(a) $q\left(z^{\lambda}\right)=\lambda^{d+1} h(z) q(z)$ and $\sum_{n=0}^{N} p_{n} \neq 0$.

(b) All roots of $h(z)$ and $q(z)$ are roots of unity.

(c) If $h(z) \in \mathbb{Q}[z]$ then $\lambda^{d+1} h(z)$ and $p_{0}^{-1} q(z)$ are monic polynomials in $\mathbb{Z}[z]$. 
Conversely, if $q(z) \mid q\left(z^{\lambda}\right)$ and $\sum_{n=0}^{N} p_{n} \neq 0$ then $f(x)$ is a normalized $\lambda$-refinable spline of degree $d$ whose mask polynomial is $h(z)=\lambda^{-d-1} q\left(z^{\lambda}\right) / q(z)$.

Proof. Assume that $f(x)$ is $\lambda$-refinable with . By (B) of Theorem 1.1 we have $\widehat{f}(\xi)=$ $\xi^{-d-1} G(\xi)$ for some trigonometic polynomial $G(\xi)$. It follows from $\widehat{f}(\lambda \xi)=H(\xi) \widehat{f}(\xi)$ where $\left.H(\xi):=h(e(-\xi)), e(t):=e^{2 \pi i t}\right)$, that $G(\lambda \xi)=\lambda d+1 H(\xi) G(\xi)$. Part (a) of the theorem now follows from $q(e(-\xi))=(-2 \pi i)^{d+1} G(\xi)$.

Assume that $q(\omega)=0$. It follow from (a) that $q\left(\omega^{\lambda}\right)=0$. This process yields roots $\left\{\omega^{\lambda^{k}}\right\}_{k \geq 0}$ for $q(z)$. But $q(z)$ has only finitely many roots so that $\omega^{\lambda^{k}}=\omega^{\lambda^{l}}$ for some $k>l$. Hence $\omega$ is a root of unity since $q(0) \neq 0$. Now $h(z)=\lambda^{-d-1} q\left(z^{\lambda}\right) / q(z)$, and thus all its roots are roots of unity as well.

We now prove (c). Without loss of generality we assume that $p_{0}=1$. So we need to prove that $q(z) \in \mathbb{Z}[z]$ and it is monic. Write $q(z)=a_{0}+a_{1} z+\cdots+a_{m} z^{m}$. Clearly $a_{0}= \pm 1 \in \mathbb{Q}$. Suppose that $a_{k}$ is irrational and $a_{j} \in \mathbb{Q}$ for $j<k$. The coefficient of the term $z^{k}$ for $\lambda^{d+1} h(z) q(z)$ is $\sum_{j=0}^{k} \lambda^{d+1} h_{j} a_{k-j}$, which is irrational since $h_{0} \neq 0$. But the first irrational coefficient in $q\left(z^{\lambda}\right)$ is of the term $z^{\lambda k}$. This is a contradiction. Hence $q(z) \in \mathbb{Q}[z]$. Now all roots of $q(z)$ are roots of unity, which means $q(z)=c q_{1}(z)$ where $q_{1}(z)$ is a product of cyclotomic polynomials, and $q_{1}(z) \in \mathbb{Z}[z]$ is monic. Note that $q(0)=(-1)^{d+1}$ and $q_{1}(0)= \pm 1$. Hence $c= \pm 1$, and $q(z) \in \mathbb{Z}[z]$. But notice that by the symmetry of refinable splines $p_{N}=p_{0}=1$. It follows that $q(z)=(z-1)^{d+1} \sum_{n=0}^{N} p_{n} z^{n}$ is monic. Also, $\lambda^{d+1} h(z)=q\left(z^{\lambda}\right) / q(z)$. So $\lambda^{d+1} h(z) \in \mathbb{Z}[z]$ and it is monic.

Conversely, suppose that $q(z) \mid q\left(z^{\lambda}\right)$ and $\sum_{n=0}^{N} p_{n} \neq 0$. We note that

$$
\widehat{B}_{d}(\xi)=(-2 \pi i)^{-d-1} \xi^{-d-1}(e(-\xi)-1)^{d+1} .
$$

Again, observe that $\widehat{f}(\xi)=(-2 \pi i)^{-d-1} \xi^{-d-1} q(e(-\xi))$, which yields $\widehat{f}(\lambda \xi)=H(\xi) \widehat{f}(\xi)$ where $H(\xi)=\lambda^{-d-1} q(e(-\lambda \xi)) / q(e(-\xi))$. Since $q(z) \mid q\left(z^{\lambda}\right), H(\xi)$ is a trigonometric polynomial. Furthermore, write $q(z)=(z-1)^{d+1} q_{1}(z) . q_{1}(z) \neq 0$ by the hypothesis. So

$$
H(0)=\lim _{z \rightarrow 1} \frac{\lambda^{-d-1} q\left(z^{\lambda}\right)}{q(z)}=\lim _{z \rightarrow 1} \frac{\lambda^{-d-1}\left(z^{\lambda}-1\right)}{z-1}=1 .
$$

Therefore $f(x)$ is $\lambda$-refinable. Furthermore the mask polynomial is $h(z)=\lambda^{-d-1} q\left(z^{\lambda}\right) / q(z) \in$ $\mathbb{R}[x]$, and $h(0)=\lambda^{-d-1} q(0) / q(0)=\lambda^{-d-1} \neq 0$. So $f(x)$ is normalized.

Lemma 3.2. Let $s, \lambda$ be positive integers. 
(a) Suppose that $\operatorname{gcd}(\lambda, s)=1$. Then $\Phi_{s}\left(z^{\lambda}\right)=\prod_{d \mid \lambda} \Phi_{d s}(z)$.

(b) Suppose that every prime factor of $\lambda$ is also a prime factor of $s$. Then $\Phi_{s}\left(z^{\lambda}\right)=$ $\Phi_{\lambda s}(z)$.

(c) Let $\lambda=\lambda_{1} \lambda_{2}$ where $\operatorname{gcd}\left(\lambda_{2}, s\right)=1$ and every prime factor of $\lambda_{1}$ is also a prime factor of $s$. Then $\Phi_{s}\left(z^{\lambda}\right)=\prod_{d \mid \lambda_{2}} \Phi_{d \lambda_{1} s}(z)$.

Proof. These are all well known results. We give a quick proof here for self-containment.

For (a), assume that $\operatorname{gcd}(\lambda, s)=1$. It is clear that any root of $\Phi_{d s}(z)$ is also a root of $\Phi_{s}\left(z^{\lambda}\right)$. So $\prod_{d \mid \lambda} \Phi_{d s}(z)$ is a factor of $\Phi_{s}\left(z^{\lambda}\right)$. On the other hand, the degree of $\prod_{d \mid \lambda} \Phi_{d s}(z)$ is

$$
\sum_{d \mid \lambda} \phi(d s)=\sum_{d \mid \lambda} \phi(d) \phi(s)=\lambda \phi(s)
$$

which is the degree of $\Phi_{s}\left(z^{\lambda}\right)$, where $\phi($.$) is the Euler's function. Both polynomials are$ monic, so they must equal. This proves (a).

For (b), it is clear that $\Phi_{\lambda s}(z) \mid \Phi_{s}\left(z^{\lambda}\right)$. The degree of $\Phi_{\lambda s}(z)$ is $\phi(\lambda s)$. Let $s=\prod_{j=1}^{m} p_{j}^{\alpha_{j}}$ be the prime factorization of $s$. By the hypothesis, $\lambda=\prod_{j=1}^{m} p_{j}^{\beta_{j}}$ where $\beta_{j} \geq 0$ (but may be 0$)$. The degree of $\Phi_{s}\left(z^{\lambda}\right)$ is

$$
\lambda \phi(s)=\lambda s \prod_{j=1}^{m}\left(1-p_{j}^{-1}\right)=\phi(\lambda s) .
$$

This proves (b).

Finally, (c) is a straightforward combination of (a) and (b).

Lemma 3.3. Let $\lambda$ be a positive integer and $q(z)=\prod_{k=1}^{m} \Phi_{b_{k}}^{\alpha_{k}}(z)$ where $\alpha_{k}>0$ and all $b_{k}$ 's are distinct. Then $q(z) \mid q\left(z^{\lambda}\right)$ if and only if for any $k$ there exists a $j=j(k)$ such that $\alpha_{j} \geq \alpha_{k}$ and $\left\langle b_{k} / \lambda\right\rangle=b_{j}$.

Proof. Observe that all $\Phi_{b_{k}}(z)$ are pairwise coprime because all $b_{k}$ 's are distinct. Hence all $\Phi_{b_{k}}\left(z^{\lambda}\right)$ are pairwise coprime.

We first prove the "if" part. To prove $q(z) \mid q\left(z^{\lambda}\right)$ we only need to prove $\Phi_{b_{k}}^{\alpha_{k}}(z) \mid q\left(z^{\lambda}\right)$ for each $k$. By the hypothesis there exists a $j$ such that $\left\langle b_{k} / \lambda\right\rangle=b_{j}$ and $\alpha_{j} \geq \alpha_{k}$. Thus it suffices to prove that $\Phi_{b_{k}}(z) \mid \Phi_{b_{j}}\left(z^{\lambda}\right)$. Note that $\frac{\lambda}{b_{k}}=\frac{c}{b_{j}}$ for some $c$ coprime to $b_{j}$ by $b_{j}=\left\langle b_{k} / \lambda\right\rangle$. Furthermore, all prime factors of $b_{j}$ are prime factors of $b_{k}$. Now let $\omega$ be a root of $\Phi_{b_{k}}(z)$, 
$\omega=e\left(\frac{a}{b_{k}}\right)$ for some $a$ coprime to $b_{k}$, where $e(t):=e^{2 \pi i t}$. Then $\omega^{\lambda}=e\left(\frac{a c}{b_{j}}\right)$. But $a$ is coprime to $b_{j}$ also, so $\omega^{\lambda}$ is a primitive $b_{j}$-th root of unity. It follows that $\Phi_{b_{k}}(z) \mid \Phi_{b_{j}}\left(z^{\lambda}\right)$.

Next we prove the "only if" part. Since $q(z) \mid q\left(z^{\lambda}\right)$ we have $\Phi_{b_{k}}^{\alpha_{k}}(z) \mid q\left(z^{\lambda}\right)$ for each $k$. Thus there exists a $j=j(k)$ such that $\Phi_{b_{k}}^{\alpha_{k}}(z) \mid \Phi_{b_{j}}^{\alpha_{j}}\left(z^{\lambda}\right)$. Since $\Phi_{b_{j}}\left(z^{\lambda}\right)$ has no multiple roots, $\alpha_{j} \geq \alpha_{k}$. Furthermore, $\Phi_{b_{k}}(z) \mid \Phi_{b_{j}}\left(z^{\lambda}\right)$. We now prove $b_{j}=\left\langle b_{k} / \lambda\right\rangle$. Let $a=\left\langle b_{k} / \lambda\right\rangle$. We have already shown in the previous part that $\Phi_{b_{k}}(z) \mid \Phi_{a}\left(z^{\lambda}\right)$. Assume that $a \neq b_{j}$. Then $\Phi_{a}\left(z^{\lambda}\right)$ and $\Phi_{b_{j}}\left(z^{\lambda}\right)$ are coprime, as stated in the beginning of the proof. Hence we cannot have $\Phi_{b_{k}}(z) \mid \Phi_{b_{j}}\left(z^{\lambda}\right)$, a contradiction. Thus $b_{j}=a=\left\langle b_{k} / \lambda\right\rangle$.

Proof of Theorem 2.2. (b) $\Rightarrow$ (a). Clearly $f(x)$ is a spline of degree $d$. Note that $\sum_{n=0}^{N} p_{n} z^{n}=p_{0} \prod_{k=2}^{m} \Phi_{b_{k}}(z)$. Hence $\sum_{n=0}^{N} p_{n}=p_{0} \prod_{k=2}^{m} \Phi_{b_{k}}(1) \neq 0$. It follows from Lemma 3.1 that $f(x)$ is normalized $\lambda$-refinable, since $q(z) \mid q\left(z^{\lambda}\right)$. Clearly $h(z)=\lambda^{-d-1} q\left(z^{\lambda}\right) / q(z) \in$ $\mathbb{Q}[z]$.

(a) $\Rightarrow$ (b). By Theorem $1.1 f(x)=\sum_{n=0}^{N} p_{n} B_{d}(x-n)$, and $q(z):=(z-1)^{d+1}\left(\sum_{n=0}^{N} p_{n} z^{n}\right)$ satisfies $q(z) \mid q\left(z^{\lambda}\right)$. It follows from Lemma 3.1 and Lemma 3.3 that $q(z)=p_{0} \prod_{k=1}^{m} \Phi_{b_{k}}^{\alpha_{k}}(z)$ where $\alpha_{k}>0$ and all $b_{k}$ 's are distinct, and for any $k$ there exists a $j=j(k)$ such that $\alpha_{j} \geq \alpha_{k}$ and $\left\langle b_{k} / \lambda\right\rangle=b_{j}$. Because $\Phi_{1}^{d+1}(z)=(z-1)^{d+1}$ is a factor of $q(z)$ we may without loss of generality assume that $b_{1}=1$, so $\alpha_{1} \geq d+1$. Now by Lemma 3.1(a) $\sum_{n=0}^{N} p_{n} \neq 0$, so $z-1$ is not a factor of $\sum_{n=0}^{N} p_{n} z^{n}$. It follows that $\alpha_{1}=d+1$, proving (a) $\Rightarrow$ (b).

Proof of Theorem 2.1. For any $a, \alpha>0$ we denote

$$
\Psi_{a, \alpha}(z)=\prod_{j=0}^{\alpha-1} \Phi_{\lambda^{j} a}(z) .
$$

(Technically $\Psi_{a, \alpha}$ also depends on $\lambda$, but we omit $\lambda$ for conciseness.) If $\operatorname{gcd}(\lambda, a)=1$ then by Lemma 3.2 ,

$$
\Psi_{a, \alpha}\left(z^{\lambda}\right)=\prod_{j=0}^{\alpha} \Phi_{\lambda^{j} a}(z)=\Psi_{a, \alpha+1}(z),
$$

and $\Psi_{a, \alpha}\left(z^{\lambda}\right) / \Psi_{a, \alpha}(z)=\Phi_{\lambda^{\alpha} a}(z)$. Furthermore if $a \neq b$ are coprime to $\lambda$ then $\lambda^{j} a \neq \lambda^{k} b$. Thus $\Psi_{a, \alpha}$ and $\Psi_{b, \beta}$ are coprime.

$(c) \Rightarrow(a)$. Observe that $q(z)=p_{0} \prod_{k=1}^{m} \Psi_{a_{k}, \alpha_{k}}(z)$. Hence $q(z) \mid q\left(z^{\lambda}\right)$. Furthermore, the multiplicity of the factor $z-1$ in $q(z)$ is precisely $d+1=\left|\left\{k: a_{k}=1\right\}\right|$. Hence 
$\sum_{n=0}^{N} p_{n} \neq 0$. It follows that $f(x)$ is a normalized $\lambda$-refinable spline of degree $d$. Since $h(z)=\lambda^{-d-1} q\left(z^{\lambda}\right) / q(z) \in \mathbb{Q}[z]$, it is also rational.

$(a) \Rightarrow(c)$. We prove it directly rather than using Theorem 2.2. By Lemma $3.1 f(x)=$ $\sum_{n=0}^{N} p_{n} B_{d}(x-n)$ with $p_{0} \neq 0, \sum_{n=0}^{N} p_{0} \neq 0$ and $q(z):=(z-1)^{d+1} \sum_{n=0}^{N} p_{n} z^{n}$ satisfying $q(z) \mid q\left(z^{\lambda}\right)$. This part of the theorem clearly follows from the following claim:

Claim: Let $g(z) \in \mathbb{Z}[z]$ be monic with $g(0) \neq 0$. If $g(z) \mid g\left(z^{\lambda}\right)$ then $g(z)$ can be expressed as a product of polynomials of the form $\Psi_{a, \alpha}(z)$ where $\operatorname{gcd}(a, \lambda)=1$ and $\alpha>0$. We recall that here $\lambda>1$ is a prime.

We prove the claim by induction. If $\operatorname{deg} g=1$, then $g(z)=z-1=\Psi_{1,1}$. Assume that the claim is true if $\operatorname{deg} g<L$, we prove it is also true if $\operatorname{deg} g=L$. The proof of Lemma 3.1 shows that all roots of $g(z)$ are roots of unity, and hence $g(z)=\prod_{j=1}^{K} \Phi_{c_{j}}^{\beta_{j}}(z)$ where $c_{j}$ 's are distinct and $\beta_{j}>0$. If all $c_{j}$ 's are coprime to $\lambda$ then $\Phi_{c_{j}}(z)=\Psi_{c_{j}, 1}(z)$, and we are done. Otherwise, without loss of generality we assume that $c_{1}=\lambda^{r} a$ with $r>0$ being the largest exponent of $\lambda$ in all $c_{j}$ 's, $\operatorname{gcd}(a, \lambda)=1$. By Lemma 3.3, $\Phi_{c_{1}}\left(z^{\lambda}\right)=\Phi_{\lambda c_{1}}(z)=\Phi_{\lambda^{r+1} a}(z)$. But $g(z) \mid g\left(z^{\lambda}\right)$, so the factor $\Phi_{c_{1}}(z)$ in $g(z)$ must be cancelled out by a factor of $\Phi_{c_{1}}(z)$ in $g\left(z^{\lambda}\right)$. Thus there exists a $j$ such that $\Phi_{c_{1}}(z) \mid \Phi_{c_{j}}\left(z^{\lambda}\right)$, yielding $c_{j}=c_{1} / \lambda=\lambda^{r-1} a$. This argument continues to show that $a, \lambda a, \ldots, \lambda^{r} a$ are all among $\left\{c_{j}\right\}$. Thus $\Psi_{a, r+1}(z) \mid g(z)$. Set $g_{1}(z)=g(z) / \Psi_{a, r+1}(z)$. Now $\operatorname{deg} g_{1}<L$. Additionally,

$$
\frac{g\left(z^{\lambda}\right)}{g(z)}=\frac{g_{1}\left(z^{\lambda}\right) \Psi_{a, r+1}\left(z^{\lambda}\right)}{g_{1}(z) \Psi_{a, r+1}(z)}=\frac{g_{1}\left(z^{\lambda}\right) \Phi_{\lambda^{r+1} a}(z)}{g_{1}(z)} .
$$

But $\Phi_{\lambda^{r+1} a}(z)$ is not a factor of $g_{1}(z)$ because we have assumed that $r$ is the largest exponent of $\lambda$ in all $c_{j}$. Hence $g_{1}(z) \mid g_{1}\left(z^{\lambda}\right)$. The induction hypothesis now applies to prove the claim. $(c) \Rightarrow(b)$. This follows directly from the fact that

$$
h(z)=\lambda^{-d-1} q\left(z^{\lambda}\right) / q(z)=h(z)=\lambda^{-d-1} \prod_{k=1}^{m} \Phi_{\lambda^{\alpha_{k}}}(z) .
$$

(b) $\Rightarrow(c)$. Write $b_{k}=\lambda^{\alpha_{k}} a_{k}$ where $\operatorname{gcd}\left(\lambda, a_{k}\right)=1$. Let $q(z)$ be given by $(2.2)$ with $p_{0} \neq 0$, and $f(x)==\sum_{n=0}^{N} p_{n} B_{d}(x-n)$. Then $f(x)$ is normalized $\lambda$-refinable with mask polynomial

$$
h(z)=\lambda^{-d-1} q\left(z^{\lambda}\right) / q(z)=h(z)=\lambda^{-d-1} \prod_{k=1}^{m} \Phi_{\lambda^{\alpha_{k}}}(z) .
$$

The proof is now complete following the uniqueness of the solution to a refinement equation. 
Proof of Theorem 2.3. (b) $\Rightarrow$ (a). By Lemma 3.1 it suffices to prove that $q(z)=$ $(z-1)^{d+1} q_{1}(z)$ satisfies $q(z) \mid q\left(z^{\lambda}\right)$. To do so we prove that if $\omega$ is a root of $q(z)$ with multiplicity $\alpha$ then $\omega$ is also a root of $q\left(z^{\lambda}\right)$ with multiplicity at least $\alpha$, which is to say that $\omega^{\lambda}$ is a root of $q(z)$ with multiplicity at least $\alpha$. If $\omega=1$ this is clearly true, so we assume that $\omega \neq 1$. It follows that $\omega$ is a root of $q_{1}(z)$. The hypothesis of (b) on $q_{1}(z)$ now implies that $\omega^{\lambda}$ is a root of $q(z)$ with multiplicity at least $\alpha$. Hence $q(z) \mid q\left(z^{\lambda}\right)$. So $f(x)$ is normalized $\lambda$-refinable, with mask polynomial $h(z)=\lambda^{-d-1} q\left(z^{\lambda}\right) / q(z)$.

$(a) \Rightarrow(b)$. By Lemma 3.1, $f(x)=\sum_{n=0}^{N} p_{n} B_{d}(x-n)$ with $p_{0} \neq 0, \sum_{n=0}^{N} p_{0} \neq 0$ and $q(z):=(z-1)^{d+1} \sum_{n=0}^{N} p_{n} z^{n}$ satisfying $q(z) \mid q\left(z^{\lambda}\right)$. All roots of $q(z)$ are roots of unity, and hence so are those of $q_{1}(z)$. Finally, the proof for $(b) \Rightarrow(a)$ clearly can be reversed, which establishes the properties for $q_{1}(z)$.

Example 3.1. With Theorem 2.3 it is rather easy to construct refinable splines that are irrational. Here is a simple example. Let $\lambda=2$. Let $\omega=\frac{1}{\sqrt{2}}+\frac{1}{\sqrt{2}} i$ be the primitive 8 -th root of unity. Let $f(x)=\sum_{n \geq 0} p_{n} B_{1}(x-n)$ with

$$
q(z)=(z-1)^{2} \sum_{n \geq 0} p_{n} z^{n}=(z-1)^{2}(z-\omega)(z-\bar{\omega})(z-i)(z+i)(z+1) .
$$

Then $q(z)$ satisfies $q(z) \mid q\left(z^{2}\right)$ by Theorem 2.3 , which can also be checked directly. The spline $f(x)$ is given by

$$
\begin{aligned}
f(x)= & B_{1}(x)+(1-\sqrt{2}) B_{1}(x-1)+(2-\sqrt{2}) B_{1}(x-2)+ \\
& (2-\sqrt{2}) B_{1}(x-3)+(1-\sqrt{2}) B_{1}(x-4)+B_{1}(x-5),
\end{aligned}
$$

and the mask polynomial for $f(x)$ is

$$
h(z)=\frac{q\left(z^{2}\right)}{4 q(z)}=\frac{1}{4}(z+1)\left(z^{2}+\sqrt{2} z+1\right)\left(z^{4}-\sqrt{2} z^{2}+1\right)
$$

Proof of Theorem 2.4. Observe that $\chi_{\left[0, N_{j}\right)}(x)$ is normalized $\lambda$-refinable for all integer $\lambda>1$, hence so is their convolution. Thus $f(x)$ is $\lambda$-refinable for all $\lambda>1$.

Conversely, let $f(x)$ be normalized $\lambda$-refinable for all $\lambda>1$. It was shown in [8] that $f(x)$ must be a spline. Thus $f(x)=\sum_{n=0}^{N} p_{n} B_{d}(x-n)$ where $d$ is the degree of the spline with $p_{0} \neq 0$ and $\sum_{n} p_{n} \neq 0$. Furthermore, $q(z)=(z-1)^{d+1} \sum_{n} p_{n} z^{n}$ has $q(z) \mid q\left(z^{\lambda}\right)$ for all $\lambda \in \mathbb{Z}^{+}$. Denote $F_{m}(z)=z^{m}-1$. Clearly, $F(z) \mid F_{m}\left(z^{\lambda}\right)$ for all $\lambda \in \mathbb{Z}^{+}$. We prove the following claim: 
Claim: Let $g(z) \in \mathbb{Z}[z]$ be monic and $g(0) \neq 0$. Suppose that $g(z) \mid g\left(z^{\lambda}\right)$ for all $\lambda \in \mathbb{Z}^{+}$. Then there exist $m_{0}, m_{1}, \ldots, m_{s}$ such that $g(z)=F_{m_{0}}(z) F_{m_{1}}(z) \cdots F_{m_{s}}(z)$.

We prove the claim by induction on $\operatorname{deg} g$. If $\operatorname{deg} g=1$ the claim is clearly true. Assume the claim holds for $\operatorname{deg} g<L$. Now let $\operatorname{deg} g=L$. By the previous results we know that all roots of $g$ are roots of unity. Let $\omega$ be a root of $g(z)$ which is an $m_{0}$-th root of unity, and assume further that no $m$-th root of unity is a root of $g(z)$ for all $m>m_{0}$. By the proof of Theorem 2.3, $\omega^{\lambda}$ is also a root of $g(z)$ for all $\lambda \in \mathbb{Z}^{+}$. By taking $\lambda$ to be $1,2, \ldots, m_{0}$ it follows that all $m_{0}$-th root of unity are roots of $g(z)$. Hence $F_{m_{0}}(z) \mid g(z)$. Write $g(z)=F_{m_{0}}(z) g_{1}(z)$. We have for any $\lambda>1$

$$
\frac{g\left(z^{\lambda}\right)}{g(z)}=\frac{z^{\lambda m_{0}}-1}{z^{m_{0}}-1} \cdot \frac{g_{1}\left(z^{\lambda}\right)}{g_{1}(z)}
$$

Observe that each root of $\frac{z^{\lambda m_{0}}-1}{z^{m_{0}}-1}$ is some $k$-th root of unity with $k>m_{0}$. Hence $\frac{z^{\lambda m_{0}}-1}{z^{m_{0}}-1}$ is coprime to $g_{1}(z)$. Thus $g_{1}(z) \mid g_{1}\left(z^{\lambda}\right)$. Since $\operatorname{deg} g_{1}<L$, the induction hypothesis now applies to prove the claim.

The theorem follows readily from the claim: There exist $N_{j} \in \mathbb{Z}^{+}, j=0,1, \ldots, s$ such that $q(z)=\prod_{j=0}^{s} F_{N_{j}}(z)$, and $s=d$ because the multiplicity of the factor $z-1$ in $q(z)$ is exactly $d+1$.

\section{REFERENCES}

[1] A. S. Cavaretta, W. Dahmen and C. A. Micchelli, Stationary subdivision. Mem. Amer. Math. Soc. 93 (1991), no. 453.

[2] E. Coven, A. Meyerowitz: Tiling the integers with translates of one finite set, J. Algebra 212 (1999), $161-174$.

[3] X. Dai, D.-J. Feng and Y. Wang, Classification of refinable splines, preprint, 2003.

[4] X. Dai, Q. SUn and Z. Zhang, Compactly supported both $\mathrm{m}$ and $\mathrm{n}$ refinable distributions, East Journal on Approximations 6 (2000), 201-209.

[5] I. Daubechies, Ten lectures on wavelets. CBMS-NSF Regional Conference Series in Applied Mathematics, 61. Society for Industrial and Applied Mathematics (SIAM), Philadelphia, PA, 1992.

[6] J. C. Lagarias and Y. Wang, Self-affine tiles in $\mathbb{R}^{n}$. Adv. Math. 121 (1996), 21-49.

[7] W. Lawton, S. L. Lee and Z. Shen, Characterization of compactly supported refinable splines. Adv. Comput. Math. 3 (1995), 137-145.

[8] Q. Sun and Z. Zhang, A characterization of compactly supported both $\mathrm{m}$ and $\mathrm{n}$ refinable distribution, J. Approx. Th. 99 (1999), 198-216. 
Department of Applied Mathematics, Zhejiang University of Technology, Hangzhou, 310014 , P. R. ChinA

E-mail address: Dai_xinrong@hotmail.com

Department of Mathematical Sciences, Tsinghua University, Beijing, 100084, P. R. China

E-mail address: dfeng@math.tsinghua.edu.cn

School of Mathematics, Georgia Institute of Technology, Atlanta, Georgia 30332, USA.

E-mail address: wang@math.gatech.edu 